\title{
Challenges of creating effective chimeric antigen receptors for cancer therapy
}

\section{"There will be different approaches for designing chimeric antigen receptor therapies to provide efficacy with limited toxicity and not a single 'best' design for chimeric antigen receptor therapy."}

\section{KEYWORDS: cell therapy $\approx$ chimeric antigen receptor $\approx$ gene therapy $\approx$ immunotherapy - toxicity}

The immune system has the ability to recognize and attack tumor cells based on the expression or overexpression of specific antigens, but immune cells often express receptors that poorly recognize tumor antigens. However, recombinant DNA techniques combined with knowledge of immune signaling has provided a means to design powerful new receptors, called chimeric antigen receptors (CARs), that can recognize any target molecule and activate a variety of cell effector functions at the site where the antigen is present. One of the primary challenges using CAR-based effector cells is to achieve good efficacy with limited toxicity. There are many different receptor designs that produce efficacious CAR cells, so there will be multiple paths that lead to success.

\section{Chimeric antigen receptors}

CARs consist of three basic parts: a recognition domain, a transmembrane domain and one or more signaling domains [1,2]. The recognition domain may be based on an antibody, a T-cell receptor or another receptor (e.g., NKG2D). The transmembrane domain includes an extracellular stalk region and may allow for dimerization. The signaling portion involves a protein domain that induces a primary activation signal in cells (e.g., CD3ל or FceRI $\gamma$ ) and one or more costimulatory domains. A primary signaling domain is sufficient to activate effector functions, but CARs that include costimulatory domains, such as from CD28, are much more effective in vivo [3,4]. Costimulatory domains have been used from $C D 28,4-1 B B, O X 40, C D 27$ and $I C O S$, and various combinations of signaling domains have been studied. In most cases, CARs are expressed in T cells, but NK cells are also used $[5,6]$. Several methods are employed to express CARs in cells: viral vectors, transposons and mRNA. Viruses and transposons will incorporate CARs into the cell DNA and lead to stable CAR expression, while mRNA will result in short-term CAR expression. CARs specific for CD19 containing either 4-1BB or CD28 signaling domains have shown spectacular results in chronic lymphocytic leukemia and B-cell acute lymphoblastic leukemia patients, respectively $[7,8]$. These outstanding clinical results, where most patients had a complete response and remain tumor-free, admittedly in only a few patients, have generated tremendous excitement for CAR cell therapy. Pharmaceutical, biotechnology and venture capital companies have begun to make significant investments in CAR therapy and clearly see it as an opportunity to have a significant impact on cancer treatment.

\section{Toxicity}

There are several different ways that CAR T cells can result in unwanted toxicity. General cell infusion reactions can occur upon infusion of a large number of activated lymphocytes, but these are often transient and manageable. More serious toxicity occurs due to activation of a large number of CAR T cells at the same time upon recognition of their target molecule on tumor cells. This can result in a cytokine storm or tumor lysis syndrome in patients. Data from a recent CAR trial in B-cell acute lymphoblastic leukemia patients targeted against CD19 supported the idea that a large tumor burden can be associated with a significant cytokine storm event [8]. These patients were given steroids, which resolved the event, but steroid treatment may limit the efficacy of the CAR cells themselves. A more difficult to predict toxicity is on-target off-tumor toxicity observed with some CARs, where the target molecule is expressed on normal tissues or there is cross-reactivity with another

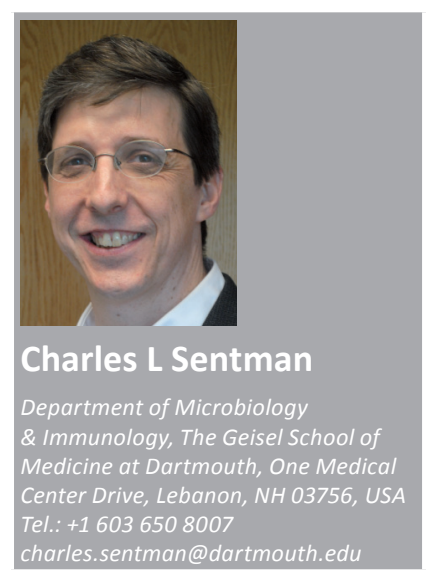

Future
Medicine $\mathrm{fS}$ 
similar antigen. In one example, two out of eight patients given MAGE-A3-specific CAR T cells developed neurotoxicity due to an unexpected cross-reactivity of the MAGE3-specific CAR with a similar peptide found on MAGE12 [9]. MAGE12 is expressed on some neurons, while MAGE3 is only expressed on tumors. These findings suggest that epitope mapping for CARs based on antibodies or T-cell receptors may be needed to help identify potential cross-reactivity. In another example, an infusion of a high number of CAR T cells $\left(10^{10}\right.$ cells) specific for ErbB2 resulted in an unexpected death, which was due to CAR recognition of low expression of ErbB2 on lung epithelium [10]. Thus, toxicity represents a significant challenge for CAR use. Toxicity may be managed through target selection, cell persistence, receptor expression and signaling design.

\section{Target selection}

One solution is for CARs to be used only against target molecules exclusively expressed on tumors or only when they are expressed on 'nonvital' cells or tissues (e.g., melanocytes). However, this would severely limit the number of possible targets to molecules such as cancer-testis antigens, mutated antigens or oncofetal antigens. These molecules are good potential targets, but many require $\mathrm{T}$-cell receptor-like recognition of specific peptides and MHC molecules, and some cancer-testis antigens are expressed by normal cells. Requirements for MHC expression on tumors and a CAR for each MHC/peptide combination limit the potential for a CAR to be widely used. The expression of a target antigen in humans is often unclear, and the extent that antigen expression may be altered in patients as a consequence of their disease or previous treatment is unknown.

\section{Cell persistance}

Toxicity is dependent on the nature of the CAR T-cell response and the number of CAR T cells infused. Low doses of CAR T cells are unlikely to result in significant toxicity; a dose can likely be found that will be safe for any CAR target, although efficacy may be reduced. CAR T cells can be found throughout the body after infusion, and due to their long-term persistence, they may find any cell expressing their target molecule and attack it. In most cases, CAR T cells are designed to remain in the host indefinitely, remaining available to attack any antigenexpressing tumor cells that they encounter. That is, CAR T cells are designed as a cell transplant.
If CAR-bearing cells were designed to survive for a limited period of time, they may be able to mediate their effector functions against the tumor, change the microenvironment and induce host antitumor immunity, while causing no or limited toxicity. Such effector cells may need to be given several doses to achieve high efficacy against tumors. This approach represents CAR cells designed as a cell drug. In fact, CAR T cells have shown rapid tumor reduction in patients [8].

\section{"Chimeric antigen receptor cell-derived cytokines can change the dynamic of the tumor microenvironment allowing host immunity to attack the tumor effectively."}

Another solution is to provide a suicide gene or targeting molecule on the CAR cells so they can be removed quickly. An inducible caspase 9 gene has been used to rapidly clear most CAR $\mathrm{T}$ cells and resolve graft-versus-host disease [11]. CAR T cells that express a marker protein, such as CD20 or truncated EGF receptor, can be targeted with an antibody as a means to rapidly eliminate them [12]. High-dose steroids have been used successfully, although this treatment also inhibits any host immune response that may have been activated by CAR T cells.

\section{Receptor expression}

It is possible to limit CAR expression on cells through the use of mRNA. Electroporation of capped mRNA results in high CAR expression, but the expression is lost as the CAR cells proliferate [13]. After several days or cell divisions, the receptor expression is low enough due to protein and mRNA turnover that the cells become ineffective. Drug-inducible CAR expression could be another method to regulate the expression of CARs on cells. CAR induction can occur in vitro or in vivo for a limited period of time. As the drug disappears, the CAR would be removed from the cell based on turnover of the receptor protein and mRNA. Moreover, it is unclear if high CAR expression is ideal. Lower expression of a CAR or a CAR with lower affinity for the target molecule may be an approach to recognize high antigen-expressing tumor cells, but not normal cells with low antigen expression. Recently, a study using two CARs, one with low affinity for antigen and another for costimulation, demonstrated selectivity for tumor cells that expressed both targeted antigens [14]. Thus, regulating receptor affinity or expression may allow selective CAR targeting of tumors. 


\section{Signaling design}

Cytokine production by CAR T cells can activate host immunity and likely represents a key element as to why these effector cells are so successful [15-17]. Efficacy is not only the result of CAR cells killing tumor cells in different tissues. Cytokines such as IFN- $\gamma$, GM-CSF and chemokines from CAR cells recruit and activate a variety of host immune cells to modulate the tumor microenvironment and disrupt tumor growth [18]. CAR cell-derived cytokines can change the dynamic of the tumor microenvironment allowing host immunity to attack the tumor effectively [19]. The design of CARs with different combinations of signaling domains may be used to promote the expression of certain cytokines but not others. This active area of research should allow the development of designer $T$ cells with uses within selected microenvironments.

\section{Conclusion}

CAR T-cell immunotherapy has demonstrated remarkable efficacy in cancer patients. However, there remain challenges for further development. It is not clear that high receptor expression, high

\section{References}

1 Sadelain M, Brentjens R, Riviere I. The basic principles of chimeric antigen receptor design. Cancer Discov. 3(4), 388-398 (2013).

2 Park TS, Rosenberg SA, Morgan RA. Treating cancer with genetically engineered $\mathrm{T}$ cells. Trends Biotechnol. 29(11), 550-557 (2011).

3 Carpenito C, Milone MC, Hassan R et al. Control of large, established tumor xenografts with genetically retargeted human $\mathrm{T}$ cells containing CD28 and CD137 domains. Proc. Natl Acad. Sci. USA 106(9), 3360-3365 (2009).

4 Savoldo B, Ramos CA, Liu E et al. CD28 costimulation improves expansion and persistence of chimeric antigen receptormodified T cells in lymphoma patients. J. Clin. Invest. 121(5), 1822-1826 (2011).

5 Kruschinski A, Moosmann A, Poschke I et al. Engineering antigen-specific primary human NK cells against HER-2 positive carcinomas. Proc. Natl Acad. Sci. USA 105(45), 17481-17486 (2008).

6 Chang YH, Connolly J, Shimasaki N, Mimura K, Kono K, Campana D. A chimeric receptor with NKG2D specificity enhances natural killer cell activation and killing of tumor cells. Cancer Res. 73(6), 1777-1786 (2013).

7 Kalos M, Levine BL, Porter DL et al. T cells with chimeric antigen receptors have potent antitumor effects and can establish memory in

affinity binding, large expansion in vivo and long-term persistence will always be desirable properties in CAR effector cells. There will be different approaches for designing CAR therapies to provide efficacy with limited toxicity and not a single 'best' design for CAR therapy.

\section{Disclaimer}

The views in this paper reflect the author's opinion and do not necessarily reflect the opinions of the NIH.

\section{Financial \& competing interests disclosure}

$C L$ Sentman's work is supported in part by grants from the NIH (CA130911, CA164178 and HL099217). The chimeric NKG2D technology developed by CL Sentman is licensed to Celdara Medical LLC. They are developing the technology for clinical use, for which he receives compensation. This arrangement is under compliance with guidelines from Dartmouth College (NH, USA). The author has no other relevant affiliations or financial involvement with any organization or entity with a financial interest in or financial conflict with the subject matter or materials discussed in the manuscript apart from those disclosed.

No writing assistance was utilized in the production of this manuscript.

patients with advanced leukemia. Sci. Transl. Med. 3(95), $95 \mathrm{ra} 73$ (2011)

8 Brentjens RJ, Davila ML, Riviere I et al. CD19-targeted $T$ cells rapidly induce molecular remissions in adults with chemotherapy-refractory acute lymphoblastic leukemia. Sci. Transl. Med. 5(177), 177ra138 (2013).

9 Morgan RA, Chinnasamy N, Abate-Daga D et al. Cancer regression and neurological toxicity following anti-MAGE-A3 TCR gene therapy. J. Immunother. 36(2), 133-151 (2013).

10 Morgan RA, Yang JC, Kitano M, Dudley ME, Laurencot CM, Rosenberg SA. Case report of a serious adverse event following the administration of $\mathrm{T}$ cells transduced with a chimeric antigen receptor recognizing ErbB2. Mol. Ther. 18(4), 843-851 (2010).

11 Di Stasi A, Tey SK, Dotti G et al. Inducible apoptosis as a safety switch for adoptive cell therapy. N. Engl. J. Med. 365(18), 1673-1683 (2011).

12 Wang X, Chang WC, Wong CW et al. A transgene-encoded cell surface polypeptide for selection, in vivo tracking, and ablation of engineered cells. Blood 118(5), 1255-1263 (2011).

13 Barrett DM, Zhao Y, Liu X et al. Treatment of advanced leukemia in mice with mRNA engineered T cells. Hum. Gene Ther. 22(12), 1575-1586 (2011).
14 Kloss CC, Condomines M, Cartellieri M, Bachmann M, Sadelain M. Combinatorial antigen recognition with balanced signaling promotes selective tumor eradication by engineered T cells. Nat. Biotechnol. 31(1), 71-75 (2013).

15 Zhang T, Barber A, Sentman CL. Chimeric NKG2D modified $T$ cells inhibit systemic

T-cell lymphoma growth in a manner involving multiple cytokines and cytotoxic pathways. Cancer Res. 67(22), 11029-11036 (2007).

16 Barber A, Zhang T, Sentman CL. Immunotherapy with chimeric NKG2D receptors leads to long-term tumor-free survival and development of host antitumor immunity in murine ovarian cancer. J. Immunol. 180(1), 72-78 (2008).

17 Pegram HJ, Lee JC, Hayman EG et al. Tumor-targeted $\mathrm{T}$ cells modified to secrete IL-12 eradicate systemic tumors without need for prior conditioning. Blood 119(18), 4133-4141 (2012).

18 Spear P, Barber A, Rynda-Apple A, Sentman CL. Chimeric antigen receptor T cells shape myeloid cell function within the tumor microenvironment through IFN- $\gamma$ and GM-CSF. J. Immunol. 188(12), 6389-6398 (2012).

19 Spear P, Barber A, Rynda-Apple A, Sentman CL. NKG2D CAR T-cell therapy inhibits the growth of NKG2D ligand heterogeneous tumors. Immunol. Cell Biol. 91(6), 435-440 (2013). 